\title{
Three decades of progress from surgery to medical therapy for isolated neuroaxis BRAF V600E-positive Langerhans cell histiocytosis management: illustrative case
}

\author{
Nallammai Muthiah, BS, ${ }^{1}$ Kamil W. Nowicki, MD, PhD, ${ }^{1}$ Jennifer L. Picarsic, MD, ${ }^{2}$ Michael P. D'Angelo, MD, ${ }^{3}$ \\ Daniel F. Marker, MD, PhD, ${ }^{4}$ Edward G. Andrews, MD, ${ }^{1}$ Edward A. Monaco III, MD, PhD, ${ }^{5}$ and Ajay Niranjan, $\mathrm{MD}^{1}$
}

Departments of ${ }^{1}$ Neurological Surgery and ${ }^{4}$ Pathology, University of Pittsburgh School of Medicine, Pittsburgh, Pennsylvania; ${ }^{2}$ Division of Pathology and Laboratory Medicine, Cincinnati Children's Hospital Medical Center, Cincinnati, Ohio; ${ }^{3}$ Department of Cardiothoracic Surgery, New York University Langone School of Medicine, New York, New York; and ${ }^{5}$ Department of Neurological Surgery, Geisinger Commonwealth School of Medicine, Danville, Pennsylvania

\begin{abstract}
BACKGROUND "Langerhans cell histiocytosis" $(\mathrm{LCH})$ is a term that encompasses single-system or multisystem disorders traditionally characterized by a proliferation of clonal CD1a+/CD207+ myeloid-derived histiocytes. In most cases of LCH, mitogen-activated protein kinase (MAPK) pathway somatic mutations lead to near universal upregulation of phosphorylated extracellular signal-regulated kinase expression. The clinical manifestations of $\mathrm{LCH}$ are numerous, but bone involvement is common. Intracranial lesions, especially as isolated manifestations, are rare.

OBSERVATIONS The authors presented the case of a long-term survivor of exclusive intracranial LCH that manifested with isolated craniofacial bone and intraparenchymal central nervous system recurrences, which were managed with 3 decades of multimodal therapy. The patient was initially diagnosed with LCH at age 2 years, and the authors documented the manifestations of disease and treatment for 36 years. Most of the patient's treatment course occurred before the discovery of $B R A F \mathrm{~V} 600 \mathrm{E}$. Treatments initially consisted of chemotherapy, radiosurgery, and open resections for granulomatous LCH lesions. Into young adulthood, the patient had a minimal disease burden but still required additional radiosurgical procedures and open resections.
\end{abstract}

LESSONS Surgical treatments alleviated the patient's immediate symptoms and allowed for tumor burden control. However, surgical interventions did not cure the underlying, aggressive disease. In the current era, access to systemic MAPK inhibitor therapy for histiocytic lesions may offer improved outcomes.

https://thejns.org/doi/abs/10.3171/CASE2118

KEYWORDS Langerhans cell histiocytosis; intracranial; Gamma Knife; radiosurgery; meningioma

Langerhans cell histiocytosis (LCH) is a collective group of rare localized, multifocal, and systemic diseases that is traditionally characterized by a proliferation of clonal CD1a +/CD207+ myeloid-derived cells that belong to the mononuclear phagocytic/dendritic cell family. The diseases are known to harbor somatic mutations, most often in the mitogen-activated protein kinase (MAPK) pathway, which leads to near-universal upregulation of phosphorylated extracellular signal-regulated kinase expression. ${ }^{1}$ Pathogenesis is a frequently debated topic with an evolving understanding of $\mathrm{LCH}$ as a clonal proliferation spanning elements of neoplasia and adaptive immunity. ${ }^{2-4}$ The natural history of $\mathrm{LCH}$ is still unclear but appears in part driven by the clonal, mutated histiocyte with contribution from systemic inflammatory signaling and tissue resident factors. $\mathrm{LCH}$ is typically diagnosed in childhood, ${ }^{5-7}$ but tissue involvement can occur at any age. LCH may involve any organ system, ${ }^{6,8}$ but it has a high affinity for bone (with estimations of bony involvement ranging from $60 \%$ to $90 \%$ of cases), followed by skin, lungs, and the pituitary gland. ${ }^{4,6}$ Isolated intracranial involvement of $\mathrm{LCH}$ is rare, and all existing case reports to date involve children. ${ }^{4,5,9,10}$ Intracranial involvement is exceedingly rare, with only $5 \%$ of patients developing central nervous system (CNS) lesions. ${ }^{11,12}$ Furthermore, in children, LCH craniofacial bone involvement (i.e., mastoid, sphenoid, orbit,

ABBREVIATIONS CNS = central nervous system; CT = computed tomography; ECD = Erdheim-Chester disease; GKRS = Gamma Knife radiosurgery; $\mathrm{LCH}=$ Langerhans cell histiocytosis; MAPK = mitogen-activated protein kinase; MRI = magnetic resonance imaging.

INCLUDE WHEN CITING Published May 10, 2021; DOI: 10.3171/CASE2118.

SUBMITTED January 6, 2021. ACCEPTED January 27, 2021.

(C) 2021 The authors, CC BY-NC-ND 4.0 (http://creativecommons.org/licenses/by-nc-nd/4.0/). 

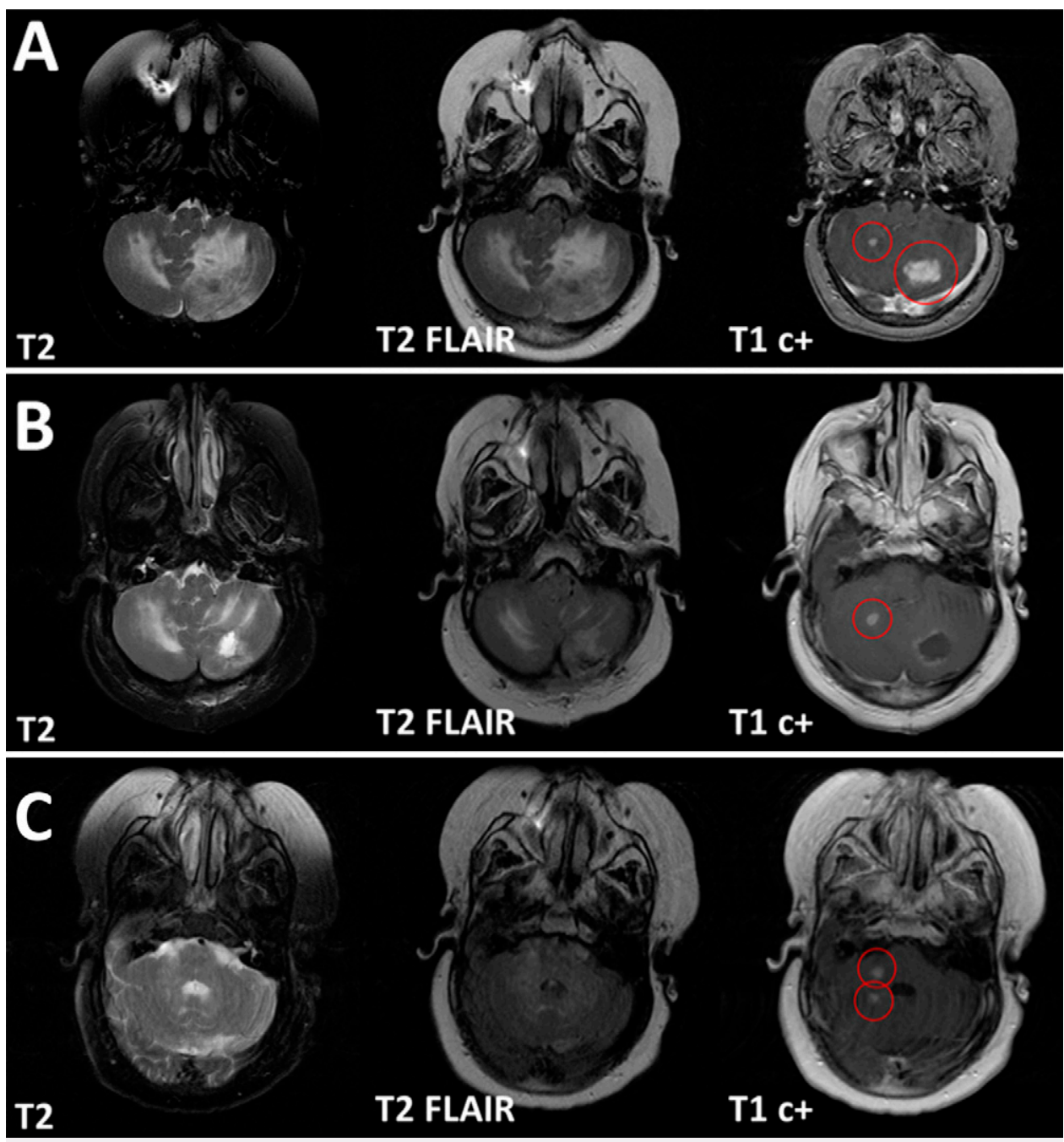

FIG. 1. Baseline MRI of the brain with and without contrast. A: MRI study obtained during the patient's first presentation to our hospital system at 24 years old (red circles indicate new lesions). B: T2, fluid-attenuated inversion recovery (FLAIR), and T1 c+ sequences after resection of cerebellar lesions via suboccipital craniotomy at 24 years old (red circle indicates remaining lesion that was primarily treated with stereotactic radiosurgery). C: New lesions in right cerebellum. MRI with and without contrast. T2, FLAIR, and T1 c+ sequences after resection of cerebellar lesions via suboccipital craniotomy at 24 years old (red circles indicate new lesions).

clivus, or temporal bone) represents CNS-risk lesions, which carry an increased risk for children to develop diabetes insipidus and/or neurodegenerative CNS LCH. ${ }^{13,14}$ The senior author (A.N.) in this study previously published findings on the use of stereotactic radiosurgery as a salvage therapy for $\mathrm{LCH}$ and reported suggested radiation doses. ${ }^{15}$ In this study, we provide a comprehensive review of the patient's medical and surgical management, detail the functional outcomes, and provide an overview of progress from surgical approach to medical therapy in the last few decades.

\section{Illustrative Case}

A 37-year-old woman with known LCH presented at our medical facility with an altered mental status. She is nonverbal and confined to a wheelchair at baseline, but her family had found her to be less outgoing and eating poorly. Her past medical history is significant for LCH diagnosed in another hospital system at age 2 years with multifocal bony intracranial CNS-risk lesions in her right mastoid, petrous, and occipital bones and right middle ear (Supplemental Fig. 1). She underwent partial resection of the mandibular and maxillary foci for debulking. She received treatment with a chemotherapeutic regimen of Decadron (a corticosteroid), mercaptopurine, methotrexate, and vinblastine per treatment protocols relevant at the time of her original diagnosis. Two years later, she presented to our facility for the first time and was found to have $\mathrm{LCH}$ recurrence of her cervical neck and right external ear canal. She was subsequently started on etoposide, prednisone, and vinblastine, with a prolonged course of treatment over 3 years. She had a third relapse 6 years later, with right temporal bone and mass involvement of the midbrain, hypothalamus, and dorsum sella that was treated with stereotactic radiosurgery. 

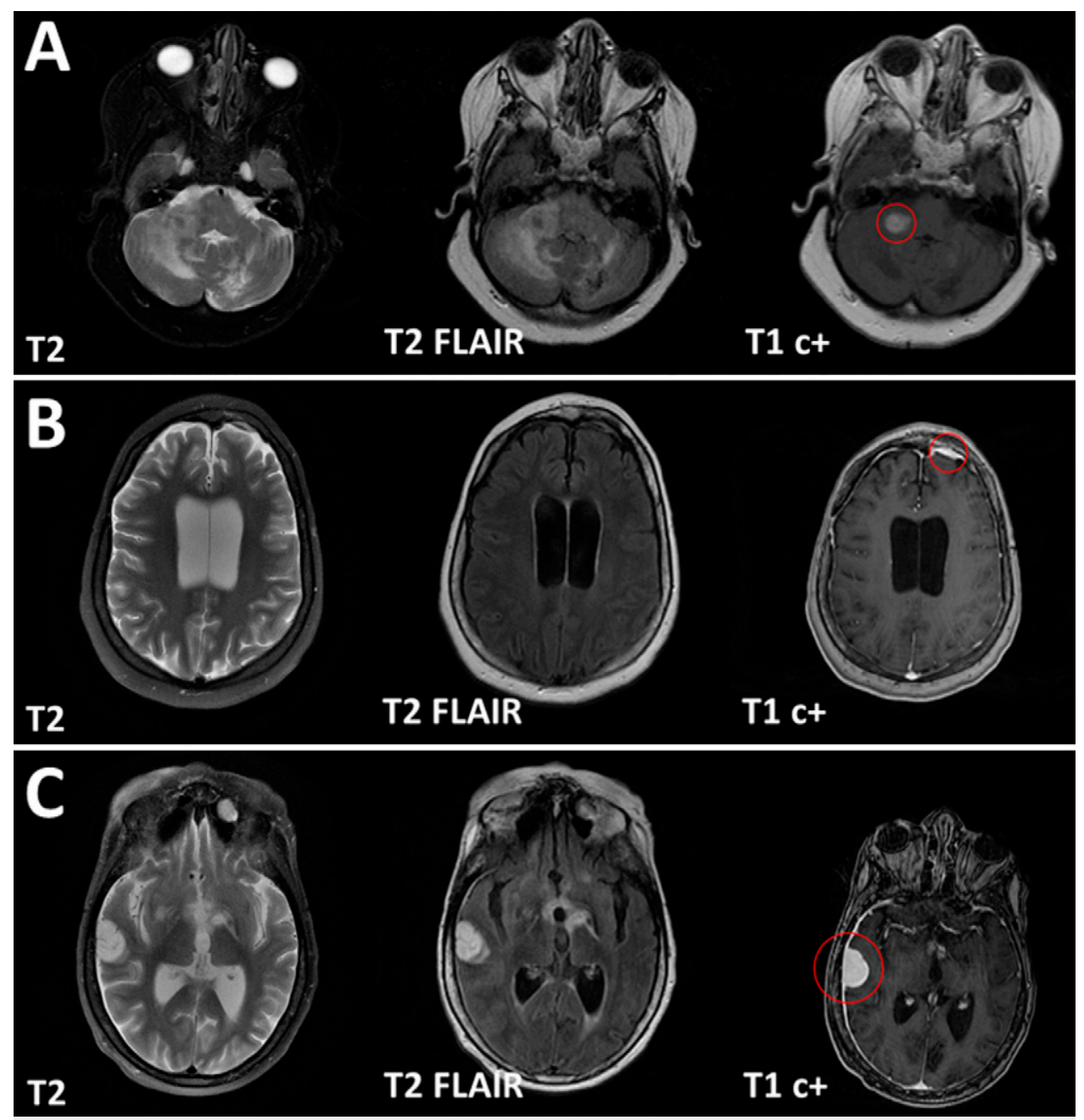

FIG. 2. Further disease progression. A: MRI with and without contrast. T2, FLAIR, and T1 c+ showing new lesion in right brachium pontis (red circle). B: MRI with and without contrast. T2, FLAIR, and T1 c+ showing new left frontal dural-based lesion (red circle). C: MRI with and without contrast. T2, FLAIR, and T1 c+ showing new parietal dural-based lesion (red circle).

During that time, the patient also underwent several radiosurgeries. During her first treatment regimen, she received 9 Gy to her right and left lateral skull and temporal bones (6 fractions in 11 days). At the end of her second treatment regimen, she received 6 Gy to the left orbit, right orbit, and left parietal areas. During her third relapse, she received 9 Gy to the right temporal bone, midbrain, and hypothalamus (5 fractions in 6 days). During the subsequent year, she received $27 \mathrm{~Gy}$ for a suprasellar tumor (15 fractions in 21 days). One year later, she underwent resection of a suprasellar and hypothalamic mass due to lesion progression. Since undergoing pituitary debulking, she has received treatment for panhypopituitarism with desmopressin and hydrocortisone.

Twenty-three years after her initial presentation, the patient presented to our facility with progressive headaches and was found to have two cerebellar lesions, one of which was causing mass effect on the fourth ventricle (Fig. 1A). A left suboccipital craniotomy for lesion resection was performed, and pathology of the lesion demonstrated $\mathrm{LCH}$. She then developed three additional lesions (one larger lesion in the left cerebellum and two smaller lesions in the right cerebellum) within 2 months (Fig. 1C). Because she previously had undergone external beam radiation therapy to the brain, the decision was made to treat the three new foci of disease with Gamma Knife (Elekta) radiosurgery (GKRS). She was treated with 13 Gy to each lesion.

The patient then received three subsequent GKRS treatments for multifocal disease: 4 years later with $14 \mathrm{~Gy}$ to the right cerebellar peduncle (Fig. 2A), the following year with 13 Gy to the left frontal dural-based lesion (Fig. 2B) and $14 \mathrm{~Gy}$ to the recurrence in right cerebellum (Supplemental Fig. 2), and 2 years later with 12 Gy to the right parietal lobe and 13 Gy to the right frontal lobe (Fig. $2 \mathrm{C})$. The following year, she presented with altered mental status that differed from her baseline. A computed tomography (CT) scan without contrast and magnetic resonance imaging (MRI) without contrast showed a large area of cerebral edema with mass effect and midline shift of $11 \mathrm{~mm}$. The patient received intravenous dexamethasone and was admitted to the intensive care unit. MRI 

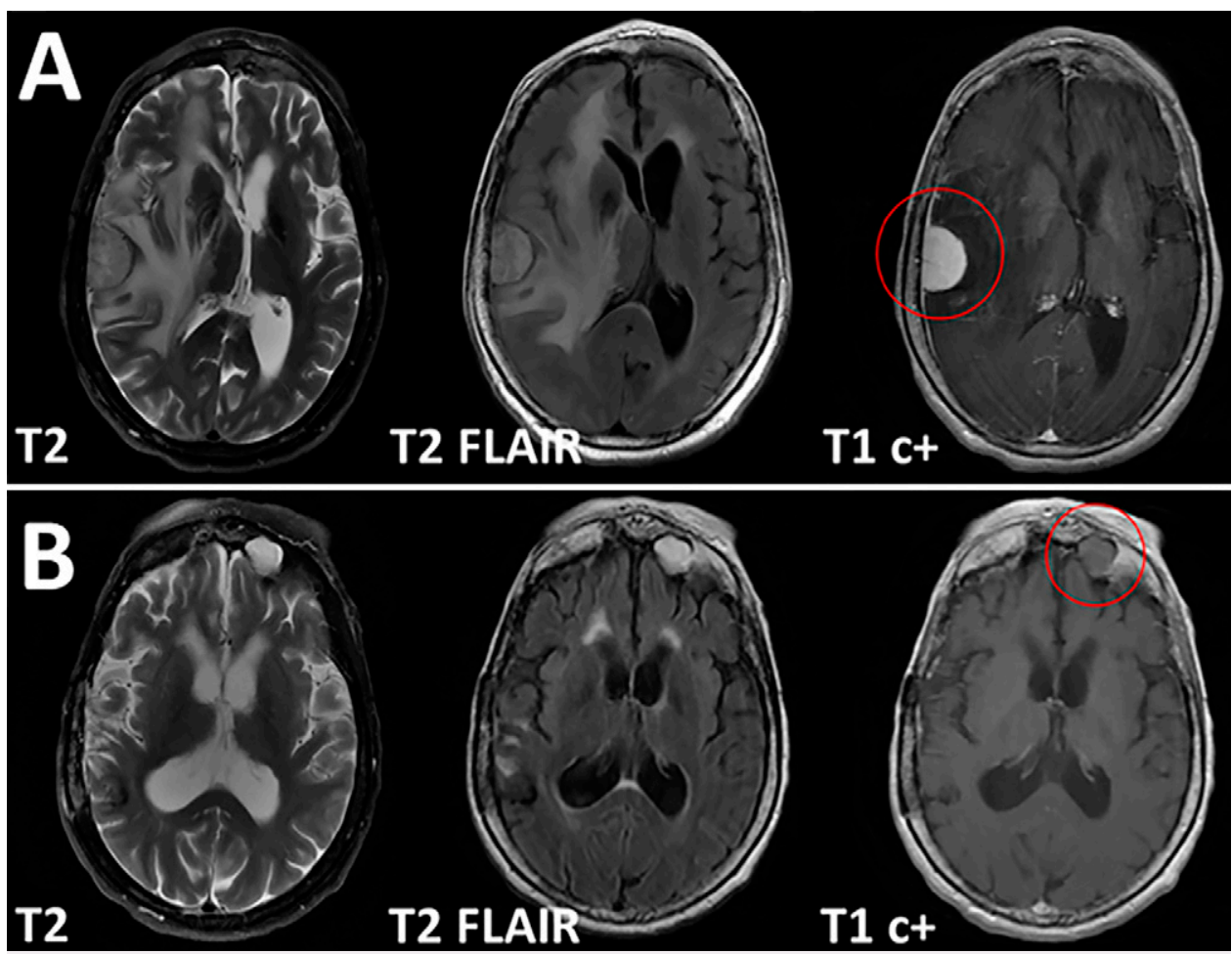

FIG. 3. A: MRI with and without contrast. T2, FLAIR, and T1 c+ showing mass effect and edema from right parietal dural lesion (red circle). B: MRI with and without contrast. T2, FLAIR, and T1 c+ showing resolved mass effect and edema 1 year after parietal meningioma resection. A left frontal meningioma was also noted (red circle).

demonstrated a leftward midline shift and partial effacement of the right ambient cistern, which had been unchanged from the previous day's CT scan but was a new development from a routine scan obtained several months earlier (Fig. 3A).

After discussion with her primary caregivers, the decision was made to resect the new lesion via a right frontotemporal craniotomy under image guidance. Gross-total resection was achieved by circumferential dissection (Fig. 3B). Small areas of hemorrhage were identified, consistent with what was seen on images. The intraoperative pathology finding was suggestive of a meningioma, with no clearly aggressive features. The final pathology report was consistent with a BRAF-negative grade I meningioma with a $\mathrm{Ki}-67$ of $15 \%$ to $20 \%$. The patient was discharged and recovered to her baseline in the following months. At her 9-month follow-up visit, MRI showed resolution of the hemorrhage and no new foci of granuloma or tumor (Fig. 4).

Subsequent review of the resected brain biopsy 23 years after initial presentation revealed small granulomatous foci of $\mathrm{LCH}$ (Fig. 5A)

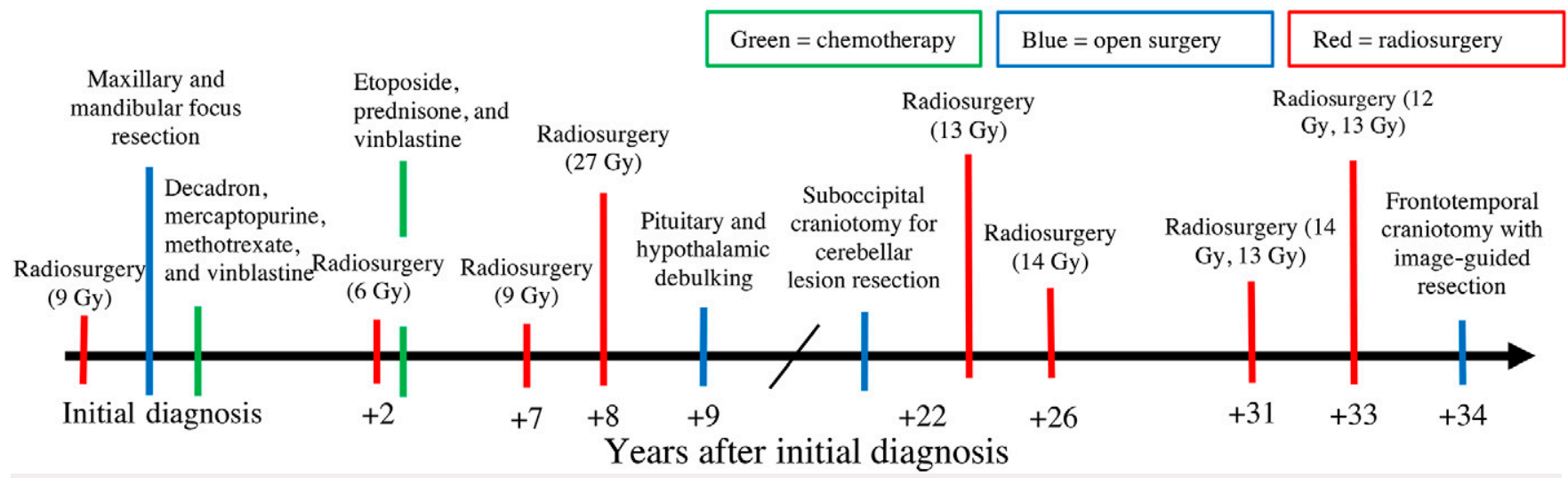

FIG. 4. Timeline summarizing the patient's surgical history. 


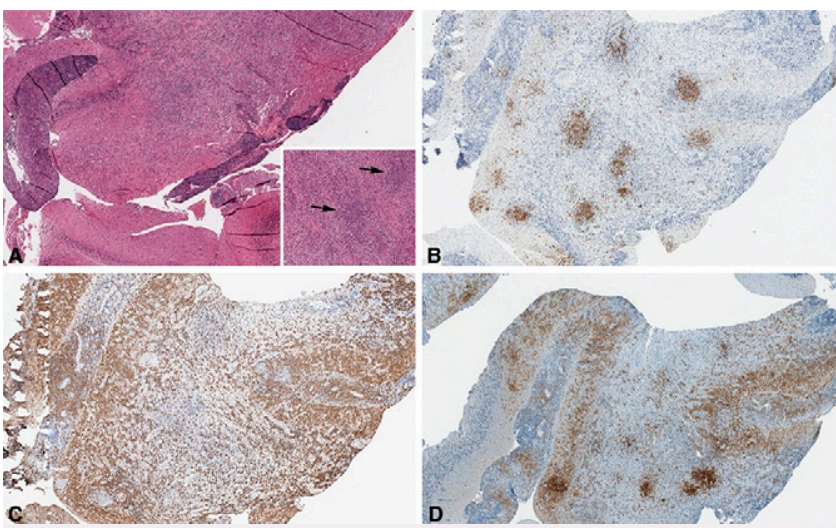

FIG. 5. Pathology of resected cerebellar lesions 23 years after initial presentation of $\mathrm{LCH}$. A: Hematoxylin and eosin staining of resected lesion showed small granulomatous foci of LCH (inset, arrows, original magnification $\times 10$ ) within a lymphohistiocytic inflammatory infiltrate. Original magnification $\times 2$. B: The $L C H$ foci were positive for $C D 207 /$ Langerin and CD1a (latter not shown) immunostains within the cerebellar parenchyma along with small collections in the inflamed leptomeninges. Original magnification $\times 2$. C: Additional immunohistochemistry shows diffuse infiltrates of plump macrophages highlighted by CD163 along with CD14 and CD68 (latter two not shown). Original magnification $\times 2$. D: The BRAF VE1 immunostain highlights the small granulomatous $\mathrm{LCH}$ nodules along with an increased number of other non$\mathrm{LCH}$ histiocytes. Original magnification $\times 2$.

highlighted by CD1a and CD207 (Fig. 5B) immunostains in the parenchyma with perivascular distribution and small infiltrating collections in the leptomeninges (Fig. 5A and B). However, the bulk of the tumorous parenchymal mass was composed of a diffusely infiltrative fibrovascular inflammatory process that contained CD163+ (Fig. 5C), CD68+, and CD14+ macrophages. Also intermixed in the inflammatory process were plasma cells, numerous $\mathrm{CD} 3+\mathrm{T}$ cells, and occasional aggregates of $\mathrm{CD} 20+\mathrm{B}$ cells. The parenchyma showed significant architectural destruction with neuronal and axonal loss and gliosis. Subsequent staining using the BRAF VE1 immunohistochemical stain showed that lesional VE1+ mutated histiocytes were present in the small granulomatous $\mathrm{LCH}$ foci and in the leptomeninges, and they were infiltrating throughout the cerebellar parenchyma with greater density than the CD1a and CD207 population (Fig. 5D). The diffuse, strong cytoplasmic expression for the BRAF VE1 immunostain was correlated with subsequent molecular polymerase chain reaction testing that confirmed the BRAF V600E mutation.

\section{Discussion}

In this study, we present a unique case that documents intracranial $\mathrm{LCH}$ progression with CNS-risk site involvement from early childhood diagnosis to adulthood, with a complex multimodal treatment and neurosurgical history. During the initial course of our patient's disease, she was placed on two separate chemotherapeutic regimens with disease recurrence. At 2 years of age, she started a course of Decadron, mercaptopurine, methotrexate, and vinblastine. Two years later, she started another regimen with etoposide, prednisone, and vinblastine.
The current LCH III regimen of corticosteroids, vinblastine, and mercaptopurine has been standard therapy for children with highrisk multisystem $\mathrm{LCH},{ }^{1,16,17}$ but this treatment regimen fails to cure more than $50 \%$ of cases in children with $\mathrm{LCH}^{18}$ Nucleoside analogs and bone marrow transplantation may be considered "salvage therapy"1 for patients whose disease is refractory to the standard treatment. In a recent international trial of 27 patients, cladribine and cytarabine were shown to be effective, but toxic, agents for patients with refractory multisystem $\mathrm{LCH}^{19}$

Historically, when chemotherapy was not indicated, radiosurgery and resection for $\mathrm{LCH}$ lesions were considered. Outcomes for radiosurgery versus resection in $\mathrm{LCH}$ lesions seem to depend at least partially on the location of the lesion. ${ }^{20}$ For example, Laird et al. performed a retrospective analysis of 39 patients with $\mathrm{LCH}$ and found that those with only bony involvement showed no 3-year recurrence after radiosurgery whereas $63 \%$ of patients with nonbone lesions experienced recurrence within 3 years. ${ }^{21}$ In the current era of histiocytosis, radiotherapy is a less favored treatment option for all forms of $\mathrm{LCH}$. Specifically, cytarabine is believed to be the most effective treatment in most patients, especially against neurodegenerative CNS LCH, with vinblastine/prednisone therapy being the second frontline regimen. ${ }^{22}$ However, a direct comparison between different chemotherapeutic regimens for $\mathrm{LCH}$ has not been performed. ${ }^{22}$

\section{Observations}

This case illustrates how the evolving understanding of the pathogenesis of disease drives medical management. Our patient's clinical management would have been drastically different had she been diagnosed as a child in the current era. She was treated based on the standard of care at that time, when far less was understood about $\mathrm{LCH}$, including its most common molecular alteration in the BRAF mutation. ${ }^{1}$ Furthermore, radiosurgery is no longer considered a major component of LCH therapy. Allen et al. published a risk-stratified algorithm in 2015 for the management of LCH based on organ system involvement. ${ }^{22}$ Based on that approach to management, our patient would have undergone a similar chemotherapeutic regimen (including vincristine, prednisone, cladribine, cytarabine, and/or clofarabine) for 12 months, with monitoring of drug toxicity; positron emission tomography, $\mathrm{CT}$, and MRI; and close attention to the development of diabetes insipidus or pituitary damage. ${ }^{22}$ Neurodegeneration also has been associated with intracranial $\mathrm{LCH}$, and it would have been specifically monitored and treated aggressively as soon as symptoms arose. ${ }^{22,23}$ Finally, she likely would have undergone genotyping for BRAFIMAP2K1 from a tumor biopsy and had her blood sampled to identify the $B R A F$ V600E mutation via quantitative polymerase chain reaction. $^{22}$

\section{Lessons}

With advances in the understanding of $\mathrm{LCH}$ as a clonal disease process with frequent MAPK pathway gene alterations, targeted oral inhibitor therapies have become more prevalent, especially in cases of aggressive and refractory disease. In recent years, the $B R A F$ V600E mutation in children with $\mathrm{LCH}$ has been correlated with worse prognosis and increased replase, ${ }^{24}$ as noted in our patient. The bulk of treatment and permanent consequences in this case occurred before the discovery of the BRAF V600E mutation in $\mathrm{LCH}$. Cases such as this one with refractory and aggressive disease appear to benefit from BRAF inhibitors in both the short term and long term. ${ }^{25,26}$ The recent VE-BASKET study of adult patients 
with histiocytic lesions showed that vemurafenib was associated with a $62 \%$ overall response rate and a progression-free survival rate of $86 \%{ }^{25}$ It is important to note, however, that this study was a nonrandomized retrospective analysis that included 26 patients with either LCH or the closely related condition Erdheim-Chester disease $(E C D)^{27}$ over the course of 2 years. There is a paucity of data to demonstrate the long-term safety and efficacy of BRAF and MAPK inhibitors in pediatric patients with $\mathrm{LCH}$; however, early results are promising. ${ }^{28,29}$ Specifically, patients with a neurodegenerative form of $\mathrm{LCH}$ and $B R A F \mathrm{~V} 600 \mathrm{E}+$ infiltrates have been shown to benefit from inhibitor therapy before significant glial scarring and permanent consequences occur. ${ }^{26}$

ECD is often thought of as having a similar disease spectrum to LCH: a myeloid inflammatory neoplasm driven by molecular alterations that upregulate the MAPK pathway. ECD is a rare form of non$\mathrm{LCH}$ with a wide range of symptoms, from localized, asymptomatic bony lesions to multiorgan involvement. ${ }^{30}$ Treatment advances for ECD have tended to follow treatments for $\mathrm{LCH}$ and vice versa. ${ }^{31}$ For example, the BRAF V600E mutation is estimated to occur in more than $50 \%$ of patients who have ECD. ${ }^{27}$ Other chemotherapeutic agents, specifically interferon- $\alpha$, have proved to be reliable and effective treatments for patients with ECD, especially patients with CNS involvment. ${ }^{27,32} \mathrm{~A}$ better understanding of the pathophysiology of either of these diseases may benefit targeted treatment for both.

Our patient's most recent right convexity lesion (Fig. 3A) was originally believed to be a focus of recurrent LCH. However, the final pathology revealed a grade I meningioma potentially associated with our patient's exposure to fractionated radiation therapy during childhood. There were no BRAF-positive neoplastic cells in the meningioma (not shown). Our patient's case also elucidates potential long-term risks of radiosurgical treatment, even when treatments are optimally administered. For our patient, radiosurgery only controlled her recurrences without significantly managing her underlying disease.

Our patient did not have extracranial bony involvement; she presented with isolated craniofacial and CNS involvement. Holbrook et al. presented one such case of a child with LCH that involved the intradural-extramedullary spinal cord without involving bone. ${ }^{5}$ Their patient presented with symptoms of compression and was ultimately found to have LCH involving the posterior cervical dura. The differential diagnosis for a posterior cervical mass in a child is broad (e.g., dermoid, epidermoid, lipoma, ependymoma, neuroblastoma, early-presenting meningioma, etc.). Their patient underwent successful treatment with steroids, surgery, and radiation. ${ }^{5}$ Although records of lesion recurrence and molecular status are not available for the patient discussed by $\mathrm{Hol}-$ brook et al., our patient's pattern of recurrence is interesting because it occurred intracranially. Although lesion recurrence in $\mathrm{LCH}$ seems to have a predilection for previously affected sites, it is unclear why only certain sites are involved. It may be related to the timing of the acquired mutation in the differentiation lineage of the neoplastic driver cell, with resident tissue factors playing a contributory role. ${ }^{1}$

Ultimately, LCH is a rare, localized, multifocal, and systemic disease that often presents in childhood. Cases such as the one involving our patient that present with the BRAF V600E mutation are associated with a more aggressive disease course with relapses and permanent consequences. We discuss an adult patient with $\mathrm{LCH}$ with $B R A F$ V600E who had an aggressive and relapsing intracranial course that was managed with 3 decades of multimodal therapy. GKRS and open resection provided temporary local control and palliative treatment for her mass lesions. Open resection was preferred in the presence of mass effect or excessive edema that could not have been adequately treated with GKRS alone. However, with the current understanding of LCH pathophysiology, this historic case may have had a different outcome if targeted small molecule inhibitor therapy were available for controlling such aggressive disease at the onset.

\section{Acknowledgements}

Thank you to Mr. Chris Woods, imaging application specialist at Cincinnati Children's Hospital Medical Center (CCHMC), for figure compilation.

\section{References}

1. Allen $\mathrm{CE}$, Merad M, McClain KL. Langerhans-cell histiocytosis. $N$ Engl J Med. 2018;379(9):856-868.

2. Abla O, Egeler RM, Weitzman S. Langerhans cell histiocytosis: current concepts and treatments. Cancer Treat Rev. 2010;36(4):354-359.

3. Harmon CM, Brown N. Langerhans cell histiocytosis: a clinicopathologic review and molecular pathogenetic update. Arch Pathol Lab Med. 2015;139(10):1211-1214.

4. Lam S, Reddy GD, Mayer R, et al. Eosinophilic granuloma/Langerhans cell histiocytosis: pediatric neurosurgery update. Surg Neurol Int. 2015;6(suppl 17):S435-S439.

5. Holbrook TJ, Fogo A, Smith HP. Histiocytosis X involving the cervical dura. Childs Nerv Syst. 1987;3(1):50-52.

6. Kasper EM, Aguirre-Padilla DH, Alter RY, Anderson M. Histiocytosis $\mathrm{X}$ : characteristics, behavior, and treatments as illustrated in a case series. Surg Neurol Int. 2011;2:57.

7. Willman CL, Busque L, Griffith BB, et al. Langerhans'-cell histiocytosis (histiocytosis X)-a clonal proliferative disease. N Engl J Med. 1994;331(3):154-160.

8. Aruna DR, Pushpalatha G, Galgali S, Prashanthy. Langerhans cell histiocytosis. J Indian Soc Periodontol. 2011;15(3):276-279.

9. Baba T, Ibayashi Y, Morimoto S, et al. A case of dural type of histiocytosis $X$ presenting as a mass lesion in the tentorium cerebelli. Article in Japanese. No Shinkei Geka. 1994;22(5):471-476.

10. Zhu M, Yu BB, Zhai JL, Sun G. Case of Langerhans cell histiocytosis that mimics meningioma in CT and MRI. J Korean Neurosurg Soc. 2016;59(2):165-167.

11. Tian Y, Wang J, Ge Jz, et al. Intracranial Rosai-Dorfman disease mimicking multiple meningiomas in a child: a case report and review of the literature. Childs Nerv Syst. 2015;31(2):317-323.

12. Wang $C$, Zou $Y$, Zeng $Q$, et al. Isolated Rosai-Dorfman disease with craniocervical junction involvement in the foramen magnum: a case report. Medicine (Baltimore). 2019;98(40):e17433.

13. Grois N, Pötschger U, Prosch $\mathrm{H}$, et al. Risk factors for diabetes insipidus in Langerhans cell histiocytosis. Pediatr Blood Cancer. 2006;46(2):228-233.

14. Grois N, Fahrner B, Arceci RJ, et al. Central nervous system disease in Langerhans cell histiocytosis. J Pediatr. 2010;156(6):873-881.e1.

15. Faramand A, Niranjan A, Flickinger J, et al. Salvage Gamma Knife stereotactic radiosurgery for recurrent intracranial Langerhans cell histiocytosis: a 36-year saga. World Neurosurg. 2020;144:205-208.

16. Tazi A, Lorillon G, Haroche J, et al. Vinblastine chemotherapy in adult patients with Langerhans cell histiocytosis: a multicenter retrospective study. Orphanet J Rare Dis. 2017;12(1):95.

17. Cazzolla AP, Troiano G, Zhurakivska K, et al. Langerhans cell histiocytosis of the maxillae in a child treated only with chemotherapy: a case report. J Med Case Reports. 2017;11(1):130.

18. Gadner H, Minkov M, Grois N, et al. Therapy prolongation improves outcome in multisystem Langerhans cell histiocytosis. Blood. 2013; 121(25):5006-5014.

19. Donadieu J, Bernard F, van Noesel M, et al. Cladribine and cytarabine in refractory multisystem Langerhans cell histiocytosis: results of an international phase 2 study. Blood. 2015;126(12):1415-1423. 
20. Hund $E$, Steiner $H$, Jansen $O$, et al. Treatment of cerebral Langerhans cell histiocytosis. J Neurol Sci. 1999;171(2):145-152.

21. Laird J, Ma J, Chau K, et al. Outcome after radiation therapy for Langerhans cell histiocytosis is dependent on site of involvement. Int J Radiat Oncol Biol Phys. 2018;100(3):670-678.

22. Allen CE, Ladisch S, McClain KL. How I treat Langerhans cell histiocytosis. Blood. 2015;126(1):26-35.

23. Grois N, Prayer D, Prosch H, Lassmann H. Neuropathology of CNS disease in Langerhans cell histiocytosis. Brain. 2005;128(pt 4):829-838.

24. Héritier S, Emile JF, Barkaoui MA, et al. BRAF mutation correlates with high-risk Langerhans cell histiocytosis and increased resistance to first-line therapy. J Clin Oncol. 2016;34(25):3023-3030.

25. Diamond EL, Subbiah V, Lockhart AC, et al. Vemurafenib for BRAF V600-mutant Erdheim-Chester disease and Langerhans cell histiocytosis: analysis of data from the histology-independent, phase 2, open-label VE-BASKET study. JAMA Oncol. 2018;4(3):384-388.

26. McClain KL, Picarsic J, Chakraborty R, et al. CNS Langerhans cell histiocytosis: common hematopoietic origin for $\mathrm{LCH}$-associated neurodegeneration and mass lesions. Cancer. 2018;124(12):2607-2620.

27. Cives M, Simone V, Rizzo FM, et al. Erdheim-Chester disease: a systematic review. Crit Rev Oncol Hematol. 2015;95(1):1-11.

28. Eckstein OS, Visser J, Rodriguez-Galindo C, Allen CE. Clinical responses and persistent $B R A F \mathrm{~V} 600 \mathrm{E}^{+}$blood cells in children with LCH treated with MAPK pathway inhibition. Blood. 2019;133 (15):1691-1694.

29. Kieran MW, Hargrave DR, Cohen KJ, et al. Phase 1 study of dabrafenib in pediatric patients (pts) with relapsed or refractory $B R A F$ V600E high- and low-grade gliomas (HGG, LGG), Langerhans cell histiocytosis (LCH), and other solid tumors (OST). J Clin Oncol. 2015;33(15 suppl):10004-10004.

30. Haroche J, Cohen-Aubart F, Amoura Z. Erdheim-Chester disease. Blood. 2020;135(16):1311-1318.

31. Milne P, Bigley V, Bacon CM, et al. Hematopoietic origin of Langerhans cell histiocytosis and Erdheim-Chester disease in adults. Blood. 2017;130(2):167-175.
32. Yang Z, Zhao S, Zhou J, et al. Erdheim-Chester disease: a case treated with IFN- $\alpha$ monitored using plasma and urine cell-free DNA. Immunotherapy. 2020;12(6):379-387.

\section{Disclosures}

The authors report no conflict of interest concerning the materials or methods used in this study or the findings specified in this paper.

\section{Author Contributions}

Conception and design: Nowicki, Picarsic, D’Angelo, Monaco, Niranjan. Acquisition of data: Nowicki, Muthiah, Marker. Analysis and interpretation of data: Nowicki, Muthiah, Picarsic, D'Angelo, Marker, Andrews. Drafting the article: Nowicki, Muthiah, Monaco. Critically revising the article: Nowicki, Muthiah, Picarsic, Marker, Andrews, Monaco, Niranjan. Reviewed submitted version of manuscript: Nowicki, Muthiah, Picarsic, Marker, Andrews, Monaco, Niranjan. Approved the final version of the manuscript on behalf of all authors: Nowicki. Administrative/technical/material support: Muthiah, Picarsic. Study supervision: Nowicki, Niranjan.

\section{Supplemental Information}

Online-Only Content

Supplemental material is available with the online version of the article. Supplemental Figs. 1 and 2. https://thejns.org/doi/suppl/10.3171/CASE2118.

\section{Correspondence}

Kamil W. Nowicki: University of Pittsburgh, Pittsburgh, PA. nowickik@upmc.edu. 